\title{
Avaliação das atividades instrumentais da vida dilária em idosos da periferia de São Luis, Maranhão
}

\author{
Life diary instrumental activities evaluation in old people \\ in outskirts in São Luiz, Maranhão
}

\section{Resumo}

A avaliação funcional determina não só o comprometimento funcional da pessoa idosa, mas sua necessidade de auxilio. É uma tentativa sistematizada e objetiva de avaliar os níveis de funcionamento numa variedade de áreas. Buscando avaliar a dependência na realização de Atividades Instrumentais da Vida Diária (AIVD), entre idosos da periferia de São Luis-MA, desenvolvemos um estudo descritivo de abordagem quantitativa. Prevaleceram idosos independentes as AIVD (variação 64,1\% a 76,6\%), "cuidar de suas finanças" apresentou a maior prevalência de ausência de limitação $(76,6 \%)$ realizando essa tarefa sem ajuda. Entre os que apresentaram alguma dependência (parcial e total), a atividade "usar telefone" foi a mais predominante (35,9\%). Em relação como cada idoso se apresentou (in)/dependente ao total das AIVD encontramos $56,3 \%$ dos idosos independentes a todas, enquanto $43,7 \%$ se mostraram com algum grau de dependência (parcial ou total). Predominaram idosos independentes, contudo entre os dependentes a maioria é de limitação a todas, representando os senis em situação crítica de dependência, que necessitam de assistência constante e especializada, polarizado por aqueles com dependência em apenas uma AIVD. O estudo revelou um diagnóstico de incapacidade funcional e a necessidade de integração dos diferentes profissionais na promoção da saúde e no apoio à comunidade.

\section{Ahstract}

The functional evaluation will determine not only the old person's functional commitment, but his/her assistance need. It's a systematic and objective try to evaluate the levels of working in areas varieties. Searching evaluate dependence through the taking of Life Diary Instrumental Activities among (LDIA) old people of São Luís-MA outskirts community, develop a descriptive study with quality approach. Prevalence has independent old people from LDIA (variation from $64,1 \%$ to $76,6 \%)$, "To take care of your own business" presented the biggest absence limitation prevalence $(76,6 \%)$ doing this chore without help. For those ones who presented some dependence (partial and total) from LDIA, the activity "to use the phone" was the most predominant $(35,9 \%)$. In a relation to each old person presented (in )/ dependent to front of the total from LDIA we found $56,3 \%$ of the independent old people from all, while $43,7 \%$ showed some dependence degree (partial or total). Predominated independent old people, nevertheless between the dependent the majority and the limitation of all, presenting the senile in dependence critical situation, which need often and specialized assistance, polarized by those senile with dependence on only an LDIA. The study discovered a diagnostic of a functional incapacity and a joining necessity of different professionals in health promotion and supportive in community.

\section{Bruno Luciano Carneiro Alves de Oliveira ${ }^{1}$ Márcia Môniea Pereira Barros ${ }^{2}$ Vanlinda de Jesus Dias Baima ${ }^{3}$ Carlos Leonardo Figueiredo Cunha ${ }^{4}$ Alécia Maria da Silva ${ }^{5}$}

Deseritores: idoso, capacidade funcional, atividades instrumentais

Keywords: old people, funetional capacity, LDIA
1 Enfermeiro especialista em Saúde da Familia - Universidade Federal do Maranhão [UFMA]. Coordenador de Atenẹão Básię em Cururupu-MA

\section{${ }^{2}$ Assistente Social especialista em Saúde da Familia - Universidade Federal do Maranhão [UFMA] \\ 3 Enfermeira especialista em Saúde da Familia - Universidade Federal do Maranhão [UFMA]. Fiseal da Vigilâneia Sanitária de São Luis-MA \\ ${ }^{4}$ Enfermeiro Professor da Universidade Federal do Maranhão. Mestre em Saúde Materno Infantil - Universidade Federal do Maranhão [UFMA]. \\ ${ }^{5}$ Enfermeira do Saúde da Familia em Cururupu-MA. Especialista em Saúde da Familia pelo GEDECON.}

Para correspondência:

Bruno Oliveira

email: brunodeoliveirama@gmail.com 


\section{Introdução}

É consenso entre os diversos autores, que a avaliação funcional é fundamental e determinará não só o comprometimento funcional da pessoa idosa, mas sua necessidade de auxílio. Pode ser compreendida como uma tentativa sistematizada de avaliar de forma objetiva os níveis no qual uma pessoa está funcionando numa variedade de áreas utilizando diferentes habilidades ${ }^{1,2}$. Representa uma maneira de medir se uma pessoa é ou não capaz de desempenhar as atividades necessárias para cuidar de si mesmas. Caso não seja capaz, verificar se essa necessidade de ajuda é parcial, em maior ou menor grau, ou total. Usualmente, utiliza-se a avaliação no desempenho das atividades cotidianas ou atividades de vida diária ${ }^{1}$.

Nas últimas décadas, o estudo de metodologias de mensuração de funções físicas, mentais e sociais expandiu consideravelmente e uma série de outros instrumentos mais sofisticados foi desenvolvida. Em 1972, Lawton criou um modelo que classificava as AVD por seu nível de dificuldade: a) atividades básicas da vida diária (relacionadas a ações básicas do cotidiano e que suprem as necessidades fundamentais, $\mathrm{O}$ autocuidado); b) atividades instrumentais da vida diária (tarefas mais complexas, relacionadas à adaptação do indivíduo no meio ambiente, a mobilidade $)^{1}$. A Escala de Lawton de Atividades Instrumentais de Vida Diária (AIVD), utilizada em nosso estudo, está entre os mais utilizados na avaliação.

\section{Método}

Assim, com o objetivo de avaliar o grau de dependência quanto à realização de atividades instrumentais da vida diária (AIVD) dos idosos cadastrados na Estratégia Saúde da Família do Centro de Saúde pertencente à comunidade do Gapara, distrito Itaqui-Bacanga, na periferia de São Luís-MA, desenvolvemos um estudo descritivo de abordagem quantitativa.

A pesquisa foi realizada após a obtenção da autorização da Secretaria Municipal de Saúde de São Luís-MA e parecer favorável do comitê de ética do HU- UFMA, respeitando os princípios estabelecidos na Resolução n ${ }^{\circ} 196$ do $\mathrm{CNS}^{3}$ e pelo Estatuto do Idoso - Lei 10.741 de 01/10/03.

Para coleta de dados foi aplicado um questionário contendo questões fechadas, onde constaram dados sobre o autocuidado e mobilidade dos idosos. Após a coleta dos dados, as questões foram codificadas e inseridas no programa EPI$\mathrm{INFO}^{\circledR}$, versão $6.0^{4}$, segundo as variáveis de interesse do estudo pesquisadas, seguiu-se a distribuição em freqüência simples, e apresentadas e agrupadas em tabelas, seguidas de análise e discussão de acordo com a literatura pertinente.

\section{Resultados}

Em relação à avaliação da capacidade funcional dos idosos da comunidade do Gapara que compuseram nosso estudo (64 idosos) encontramos a distribuição dessa informação na tabela 1 . As AIVD são relacionadas à participação dos idosos em seu entorno social e indicam a capacidade de um indivíduo em levar uma vida independente (física e mental) dentro da comunidade ${ }^{1,5}$.

A amostra de idosos entrevistada constituiuse basicamente de idosos independentes entre às AIVD (variação de $64,1 \%$ a $76,6 \%$ ). Em relação ao grau de dependência para as AIVD, prevaleceu a condição de independentes $(56,3 \%, n=36)$. A razão de proporção entre independência/dependência foi de 1,29 vezes. Entre os dependentes $(43,7 \%, n=28)$ a maior prevalência foi de dependência a todas as AIVD $(21,4 \%, n=6)$ seguindo de 17,8\% (n=5) dependentes em apenas uma AIVD; $14,3 \%(n=4)$ se mostraram dependentes a duas AIVD; 10,7\% $(n=3)$ dos senis se mostraram dependentes a três e quatro AIVD respectivamente, o que representa o contingente de idosos em situação crítica de dependência, e que necessitam de assistência constante e relativamente especializada.

Em relação à avaliação da independência (tabela 1) as AIVD "cuidar de suas finanças" apresenta a maior prevalência de ausência de limitação $(76,6 \%, \mathrm{n}=49)$ realizando essa tarefa sem ajuda, seguido de "fazer compras", "preparar as próprias refeições" e "trabalhos manuais domésticos" (com 71,9\%, n=46, dos idosos respectivamente independentes). Enquanto "ir a locais distantes" e "tomar seus remédios na dose e horários corretos" apresentaram prevalências simultâneas $(70,3 \%, n=45)$. Entre as AIVD que apresentaram idosos menos independentes está crescentemente "usar telefone" $(64,1 \%, \mathrm{n}=41)$, seguido de "arrumar a casa" $(67,2 \%, \mathrm{n}=43)$ e finalmente "lavar e passar sua roupa" $(68,8 \%, n=44)$.

Considerando os idosos de nosso estudo que apresentaram alguma dependência (parcial e total) (Tabela 1) entre as AIVD encontramos a atividade 
"usar telefone" como a atividade mais predominante $(35,9 \%, n=23)$. Em segundo lugar surge "arrumar a casa" (32,8\%, n=21), enquanto "lavar e passar sua roupa" representa a terceira valores polarizados bem próximos de Adriano Luiz da Costa Farinasso ${ }^{6}(61,6 \%)$ e de Rosana Aparecida Andreotti e Silene Sumire Okuma ${ }^{1}$ (75\%). Em relação à maior prevalência de idosos na condição

\begin{tabular}{|c|c|c|c|c|c|c|}
\hline \multirow{2}{*}{$\begin{array}{l}\text { ATIVIDADES } \\
\text { INSTRUMENTAIS DA VIDA } \\
\text { DIÁRIA }\end{array}$} & \multicolumn{2}{|c|}{ Sem ajuda } & \multicolumn{2}{|c|}{ Com ajuda parcial } & & \\
\hline & $f$ & $\%$ & $f$ & $\%$ & $f$ & $\%$ \\
\hline Usar telefone & 41 & 64,1 & 13 & 20,3 & 10 & 15,6 \\
\hline Ir a locais distantes & 45 & 70,3 & 10 & 15,6 & 9 & 14,1 \\
\hline Fazer compras & 46 & 71,9 & 8 & 12,5 & 10 & 15,6 \\
\hline $\begin{array}{l}\text { Preparar as } \\
\text { próprias refeições }\end{array}$ & 46 & 71,9 & 7 & 10,9 & 11 & 17,2 \\
\hline Arrumar a casa & 43 & 67,2 & 9 & 14,0 & 12 & 18,8 \\
\hline $\begin{array}{l}\text { Trabalhos manuais } \\
\text { domésticos }\end{array}$ & 46 & 71,9 & 10 & 15,6 & 8 & 12,5 \\
\hline Lavar e passar sua roupa & 44 & 68,8 & 7 & 10,9 & 13 & 20,3 \\
\hline $\begin{array}{l}\text { Tomar seus remédios na dose } \\
\text { e horários corretos }\end{array}$ & 45 & 70,3 & 10 & 15,6 & 9 & 14,1 \\
\hline Cuidar de suas finanças & 49 & 76,6 & 11 & 17,1 & 4 & 6,3 \\
\hline
\end{tabular}

Fonte: Direta

Tabela 1. Distribuição das freqüências de dependência dos idosos da Comunidade do Bairro Gapara segundo à realização de atividades instrumentais da vida diária (AIVD), São Luís-Ma, 2008.

forma mais predominante $(31,2 \%, n=20)$ dos senis com alguma limitação para realizar essa atividade, seguido por "ir a locais distantes" e "tomar seus remédios na dose e horários corretos" (29,7\%, n=19) respectivamente. Simultaneamente as AIVD "fazer compras", "preparar as próprias refeições" e "trabalhos manuais domésticos" aparecem com prevalências iguais $(28,1 \%, n=18)$. Por último a atividade que exige menor limitação, alguma ajuda para realizá-la foi "cuidar de suas finanças" (23,4\%, $\mathrm{n}=15)$.

A amostra de idosos entrevistada constituiuse de uma proporção menor de idosos dependentes (parcial e total) às AIVD (variação de 23,4\% a 35,9\%). Além disso, a proporção da prevalência de dependência encontrada em nossa pesquisa apresentou média de $29,7 \%$.

\section{Discussão}

Os idosos entrevistados apresentaram-se independentes entre às AIVD em $(64,1 \%$ a $76,6 \%)$ de independentes as AIVD, João Macedo Coelho Filho e Luiz Roberto Ramos ${ }^{7}$, em pesquisa com idosos, corroboram nossa pesquisa (56,3\%) ao encontrarem dados semelhantes, (52,3\% em Fortaleza e 53,0\% em São Paulo) de idosos com autonomia total para realização das AIVDs. Além disso, encontraram uma proporção de 35\% que necessitavam de ajuda total ou parcial para realizarem até três atividades; 9,9\%, quatro a seis e $2,8 \%$, sete ou mais atividades.

Em relação à avaliação da independência as AIVD, Marcelo Cortes Néri ${ }^{8}$ em seus estudos revela que $85 \%$ dos idosos têm o controle sobre as suas próprias despesas, o que denota autonomia, dado interessante que contrasta com o senso comum, segundo o qual os idosos são dependentes. A independência financeira é um dos fatores essenciais para a autonomia e pode influenciar uma percepção mais positiva da qualidade de vida.

Os idosos entrevistados apresentaram-se numa proporção menor de idosos dependentes (parcial e total) às $\operatorname{AIVD}(23,4 \%$ a $35,9 \%)$ com valores 
polarizados bem próximos aos de Maria Rosa Mendes Fielder ${ }^{5}(37,1 \%)$, de João Macedo Coelho Filho e Luiz Roberto $\operatorname{Ramos}^{7}(35 \%)$ e de Maria Lúcia Lebrão e Rui Laureti ${ }^{9}$ (26,5\%). Além disso, a proporção da prevalência de dependência encontrada em nossa pesquisa $(29,7 \%)$ foi ligeiramente menor do que a média encontrada no Nordeste brasileiro, que foi de $35,0 \%$; fato que mostra a confiança de nossa pesquisa e a confluência de realidade que os senis encontram.

Celita Salmaso Trelha et al. ${ }^{2}$ em um estudo sobre a capacidade funcional de idosos restritos ao domicílio na cidade de Londrina (PR) verificou as AIVD "fazer compras" e "utilizar o telefone" como às atividades que obtiveram a maior porcentagem de dependência ( $53,8 \%$ e $42,3 \%$, respectivamente).

$\mathrm{Na}$ avaliação de Adriano Luiz da Costa Farinasso ${ }^{6}$, as dificuldades referidas pelos idosos na tarefa "usar telefone" não devem ser relacionadas apenas às limitações físicas dos senis. $\mathrm{O}$ rápido avanço tecnológico nas telecomunicações, com constantes mudanças nos usos dos serviços telefônicos, possivelmente, estão propiciando algum nível de dificuldade atribuída pelos idosos àquela tarefa.

No entendimento de Maria Rosa Mendes Fielder ${ }^{5}$ a escolaridade (nível de informação) e as condições de saúde influenciam na percepção da realidade e das tecnologias limitando esses idosos ao auxílio, em parte, não por incapacidade funcional, mas por falta de instrução e orientação. Enquanto a longevidade e a freqüência das doenças crônicas mostram-se fatores fortemente associados à perda da capacidade funcional ${ }^{5,10}$.

Para Maria Rosa Mendes Fielder ${ }^{5}$, a capacidade funcional pode ser definida como a capacidade que os idosos têm em manterem-se independentes. A utilização de avaliações da capacidade funcional tem a possibilidade de fornecer informações sobre o grau de dependência da pessoa idosa, os tipos de cuidados que vão ser necessários e o perfil do idoso, sendo ferramentas simples e úteis na identificação das limitações e perda da autonomia do idoso, definindo estratégias de promoção de saúde dos idosos visando retardar ou prevenir as incapacidades. Essa avaliação da rede de suporte social e da funcionalidade familiar tornase essencial para o planejamento da atenção a pessoa idosa ${ }^{2,5}$.

Consubstanciando em Maria Rosa Mendes Fielder ${ }^{5}$ através dos resultados do nosso estudo é possível concluir que a dependência compromete a autonomia total da pessoa, dificultando as relações familiares, a participação do idoso na sociedade e fragilizando a qualidade de vida.

Segundo Ana Amélia Camarano ${ }^{11}$, o grupo populacional acima de 60 anos de idade é bastante heterogêneo em decorrência do próprio processo de envelhecimento que se manifesta de modo singular em cada idoso. Assim, a determinação da capacidade funcional objetivando conhecer as limitações de cada senil produz a expectativa de construir modelos de atenção também individuais uma vez que se tem observado que a dependência (parcial ou total) parece ser regra, não exceção.

\section{Conclusões}

Os resultados encontrados mostram que houve maior prevalência dos senis independentes as AIVD. Todavia, entre os dependentes as AIVD prevaleceram os que tinham dependência a todas as essas atividades, o que representa um cenário de idosos em situação critica de dependência, e que precisam de cuidado permanente e relativamente especializado, polarizado por aqueles senis com dependência em apenas uma AIVD.

A AIVD "cuidar de suas finanças" apresentou a maior prevalência de ausência de limitação, enquanto "usar o telefone" apresentou-se nos senis mais dependentes. Corroborando nossos dados, algumas pesquisas mostram que $75 \%$ da população idosa mundial independem de outros para realizar suas tarefas cotidianas ${ }^{1 .} \mathrm{E}$ outros estudos demonstraram que nossos dados estão próximos dos dados das pesquisas deles e que a capacidade funcional surge como um conceito mais adequado para instrumentalizar e operacionalizar a atenção à saúde do idoso, sobre a ótica da saúde coletiva $^{2,5-9}$.

Assim, o presente trabalho revela um diagnóstico de incapacidade funcional apresentado pelos idosos e destaca também a necessidade de integração dos diferentes profissionais envolvidos nessa questão, com suas competências específicas e em sintonia de ações de promoção da saúde até o estabelecimento de redes de apoio a cuidados de longa duração na comunidade, buscando a manutenção do convívio em suas residências de origem evitando o risco de institucionalização. As estratégias que vislumbram esse modelo de atenção à terceira idade deverão considerar o fenômeno do envelhecimento que cresce gradualmente no Brasil ${ }^{2}$.

Esta investigação teve o objetivo de 
contribuir para o avanço do conhecimento na área de atenção ao idoso e fornecer subsídios aos elaboradores/executores de políticas públicas e sociais para atender a crescente massa de idosos e ajudar na implementação de espaços sociais de cuidado ao idoso.

\section{Referêneias}

1. Andreotti RA, Okuma SS. Validação de uma bateria de testes de atividades da vida diária para idosos fisicamente independentes. Rev. Paul. Educ. Fís., 1999; 13(1): 46-66.

2. Trelha CS, Nakaoski T, Franco SS, Dellaroza MSG, Yamada K, Cabreara M et al. Capacidade funcional de idosos restritos ao domicílio, do conjunto Ruy Virmond Carnascialli, Londrina/PR. Semina Cienc. Biol. Saúde, 2005; 26(1): 37-46.

3. Ministério da Saúde. Resolução $\mathrm{n}^{\circ}$ 196, de 10 de outubro de 1996. Aprova as diretrizes e normas regulamentadoras de pesquisas envolvendo seres humanos. Diário Oficial União. 16 out 1996.

4. Dean AG, Dean JA, Coulombier D et al. Epi Info, version 6.04: um sistema de processamento de texto, banco de dados e estatística para computadores. São Paulo: Centers of Disease Control; 2001.

5. Fielder MM. Prevalência de baixa capacidade funcional entre idosos residentes na zona urbana de Joaçaba 2003/2004. Santa Catarina: Universidade do Oeste de Santa Catarina; 2005.

6. Fielder MM. Prevalência de baixa capacidade funcional entre idosos residentes na zona urbana de Joaçaba 2003/2004. Santa Catarina: Universidade do Oeste de Santa Catarina; 2005.

7. Coelho Filho JM, Ramos LR. Epidemiologia do envelhecimento no Nordeste do Brasil: resultados de inquérito domiciliar. Rev. Saúde Pública, 1999; 33(5): 44553.

8. Néri MC. Renda, consumo e aposentadoria: evidências, atitudes e percepções. In: Néri AL. Idosos no Brasil: vivências, desafios e expectativas na terceira idade. São Paulo: Fundação P. Abramo; 2007. p. 01-27.

9. Lebrão ML, Laurenti R. Saúde, bem-estar e envelhecimento: o estudo SABE no Município de São Paulo. Rev. Bras. Epidemiol., 2005; 8(2): 127-141.

10. Karsch UM. Idosos dependentes: famílias e cuidadores. Cad. Saúde Pública, 2003; 19(3): 861-866.

11. Camarano AA. Envelhecimento da população brasileira: problema para quem? Salvador: Bahia Análise e Dados, 2001; 10 (4): 36-48. 